\title{
Tritium at the Steel Creek Landing
}

by

M. Arnett

Westinghouse Savannah River Company

Savannah River Site

Aiken, South Carolina 29808

J. D. Heffiner

P. D. Fledderman

J. W. Littrell

D. W. Hayes

M. S. Dodgen

DOE Contract No. DE-AC09-96SR18500

This paper was prepared in connection with work done under the above contract number with the U. S.

Department of Energy. By acceptance of this paper, the publisher and/or recipient acknowledges the U. S. Government's right to retain a nonexclusive, royalty-free license in and to any copyright covering this paper; along with the right to reproduce and to authorize others to reproduce all or part of the copyrighted paper.

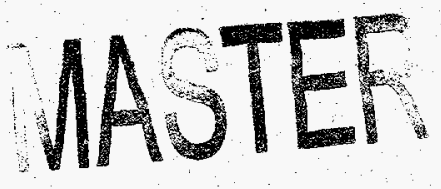

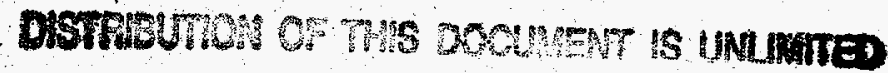




\section{DISCLAIMER}

This report was prepared as an account of work sponsored by an agency of the United States Government. Neither the United States Government nor any agency thereof, nor any of their employees, makes any warranty, express or implied, or assumes any legal liability or responsibility for the accuracy, completeness, or usefulness of any information, apparatus, product, or process disclosed, or represents that its use would not infringe privately owned rights. Reference herein to any specific commercial product, process, or service by trade name, trademark, manufacturer, or otherwise does not necessarily constitute or imply its endorsement, recommendation, or favoring by the United States Government or any agency thereof. The views and opinions of authors expressed herein do not necessarily state or reflect those of the United States Government or any agency thereof.

This report has been reproduced directly from the best available copy.

Available to DOE and DOE contractors from the Office of Scientific and Technical Information, P.O. Box 62, Oak Ridge, TN 37831; prices available from (615) 576-8401.

Available to the public from the National Technical Information Service, U.S. Department of Commerce, 5285 Port Royal Road, Springfield, VA 22161. 


\section{DISCLAIMER}

Portions of this document may be illegible electronic image products. Images are produced from the best available original document. 
ESH-EMS-98-0271

\section{'Tritium at the Steel Creek Landing}

A discussion of recent sampling results from WSRC's Environmental Monitoring Section and the South Carolina Department of Health and Environmental Control

J.D. Heffner

P.D. Fledderman

J.W. Littrell

D.W. Hayes

M.S. Dodgen 


\section{Tritium at the Steel Creek Landing}

Summary

In December 1997 and January 1998, the South Carolina Department of Health and Environmental Control (SCDHEC) collected routine weekly grab samples from the Savannah River near the Steel Creek Boat Landing. The samples from December 30 and January 5 showed atypically high tritium concentrations (around $20 \mathrm{pCi} / \mathrm{mL}$, compared with typical concentrations of 1 to $3 \mathrm{pCi} / \mathrm{mL}$ ). The Environmental Monitoring Section (EMS) of Westinghouse Savannah River Company (WSRC) investigated and found no abnormal tritium releases to the Savannah River. Portions of SCDHEC's samples were provided to EMS for confirmatory analyses, and EMS confirmed SCDHEC's results. Investigation of prior studies of the Savannah River revealed firm evidence that the elevated tritium concentrations originated in Four Mile Creek and Pen Branch and that they appeared in SCDHEC's samples as a result of (1) incomplete mixing of stream water and river water and (2) differences in the sampling locations and methods."

Details

\section{Tritium in the Savannah River}

SCDHEC's weekly sample from December 30 showed a tritium concentration of $19.6 \mathrm{pCi} / \mathrm{mL}$. The Savannah River's tritium concentration typically is 1 to $3 \mathrm{pCi} / \mathrm{mL}$.

Normally, the largest contributions of tritium in the Savannah River originate in tritiated groundwater that migrates into Four Mile Creek and Pen Branch. Concentrations in Pen Branch and Four Mile Creek typically are 100 and $200 \mathrm{pCi} / \mathrm{mL}$, respectively. In 1996, site streams transported about 8000 curies of tritium to the Savannah River. Of the 8000 curies, about 4600 curies were transported by Four Mile Creek, and about 2300 curies were transported by Pen Branch. All other streams combined transported about 1000 curies [SRS Environmental Data for 1996, WSRC-TR-97-0077, p.26). Because Four Mile Creek and Pen Branch account for most tritium in the Savannah River, they are identified as likely sources of the tritium in SCDHEC's samples of concern.

Additional smaller sources of tritium in the Savannah River include process water discharged into Beaver Dam Creek at D-Area, ETF discharges into Upper Three Runs Creek, and small amounts from PAR Pond and Steel Creek.

\section{Sampling Locations}

EMS and SCDHEC maintain networks of sampling locations. Data from multiple stations are compared routinely. EMS monitors tritium in the Savannah River at several locations and in each site tributary to the river. Sampling points are shown in figure 1. EMS sampling devices work continuously, and samples are collected and analyzed weekly. EMS samples are collected from sections of the river at which flow is . always available for sampling. SCDHEC's grab samples are taken near the river bank. When the river's water level rises, SCDHEC's sampling points recede landward.

EMS quantifies the amount of tritium discharged to each site stream, the tritium transported by each stream, and the tritium transported by the river. Any abnormal result from any single station can be confirmed or refuted by data from other stations. EMS's key sampling locations for this study are located at River Mile 120 (Highway 301 Bridge) and River Mile 140 (just downstream of the Steel Creek Mouth). Sampling stations on each site tributary contribute supporting data.

In this case, EMS evaluated samples collected from each tributary and from the Savannah River stations at the same times that SCDHEC's samples of concern were collected. None showed abnormally high tritium concentrations. Likewise, SCDHEC's weekly composite samples from the Savannah River showed no abnormally large tritium transport in the river. 
The initial interpretation was that incomplete mixing of low-tritium water in the Savannah River and hightritium water from Four Mile Creek and Pen Branch caused the water near the bank at Steel Creek Landing to show elevated tritium concentrations, while fully mixed downstream river water showed normal levels of tritium.

\section{Previous Studies}

Three previous SRS studies were identified as relevant to interpreting the observed conditions.

In March 1983, thermal infrared aerial photographs surveyed parts of the Savannah River where site tributaries discharged hot water. Conditions at the time of this survey included a high river stage and a flooded swamp. The photographs (reproduced in figure 2) show clearly that in this flow regime, waters discharged from Four Mile Creek and Pen Branch emerge into the swamp and migrate close to the river bank for several miles, then merge and mix with the main river flow downstream of Steel Creek landing.

In August 1991, thermal infrared aerial photographs were taken in the same area. Conditions at the time of this survey were low river flow, without swamp flooding. The photographs show clearly that in these conditions, waters from Four Mile Creek and Pen Branch enter the Savannah River and mix thoroughly within 2000 feet of downstream flow. These photographs are reproduced in figure 3.

In February 1990, under conditions of high river flow and flooded swamp, water samples were collected in a transect from the submerged normal riverbank location landward toward the floodstage riverbank. Analyses of these samples showed low tritium concentrations in the river channel and near the normal bank. Concentrations were low along most of the transect but were elevated for the few hundred feet of swamp nearest the temporary bank. Analytical data from this study are shown in figure 4.

\section{Current Data}

SCDHEC's weekly grab samples from 1997 occasionally show elevated tritium concentrations. The highest concentrations observed coincide with high river stage and swamp flooding. Weekly composite samples from EMS's samplers at the Highway 301 Bridge show a decreased concentration of tritium when the river flow is high. The Savannah River stage, as reported by the U.S. Army Corps of Engineers at their Jackson gauging station, is shown in figure 5. Note that the periods of highest flow in the last year occurred in March, May, and December, which coincide with times of elevated tritium concentrations in SCDHEC's weekly grab samples. SCDHEC's weekly sample results are reproduced in attachment 1 . Composite samples from the same periods show depressed tritium concentrations, as would be expected when a fairly constant source of tritium in SRS streams is diluted by high river flow. Results from 1997 composite sample analyses are shown in table 1 .

\section{Conclusions}

Historical studies have shown two distinctly different patterns in the mixing of water from the Savannah River with water from two tributaries, Four Mile Creek and Pen Branch. It is expected that the mixing pattern shown in the thermal infrared aerial photographs applies to tritiated water in the tributaries, as well as to hot water. This conclusion was confirmed with a sampling study in 1990.

When the Savannah River stage is high, the swampy area along SRS is flooded, with average water depth of a few feet, and with slow low-turbulence flow. Under these conditions, Four Mile Creek and Pen Branch water enters the swamp and stays close to the bank. This water does not disperse and mix fully with Savannah River water until it moves downstream past the Steel Creek Landing.

When the Savannah River stage is low, Four Mile Creek flows through a well-defined mouth directly into the river channel and mixes thoroughly with river water within a few thousand feet.

Regardless of river stage, the Savannah River at the Highway 301 Bridge is fully mixed with all tributaries that cross SRS. 
SCDHEC grab samples collected at the water's edge during high-flow conditions would be expected to reflect Four Mile Creek and Pen Branch water that has not been mixed completely with Savannah River water. The incomplete mixing leads to tritium concentrations in those samples that do not represent tritium concentrations in the main flow of the Savannah River. December 1997 samples from downstream and from the two tributaries did not show unusual tritium levels. 
Figure 1 Sampling Locations

Reproduced from SRS Environmental Data for 1996, WSRC-TR-97-0077

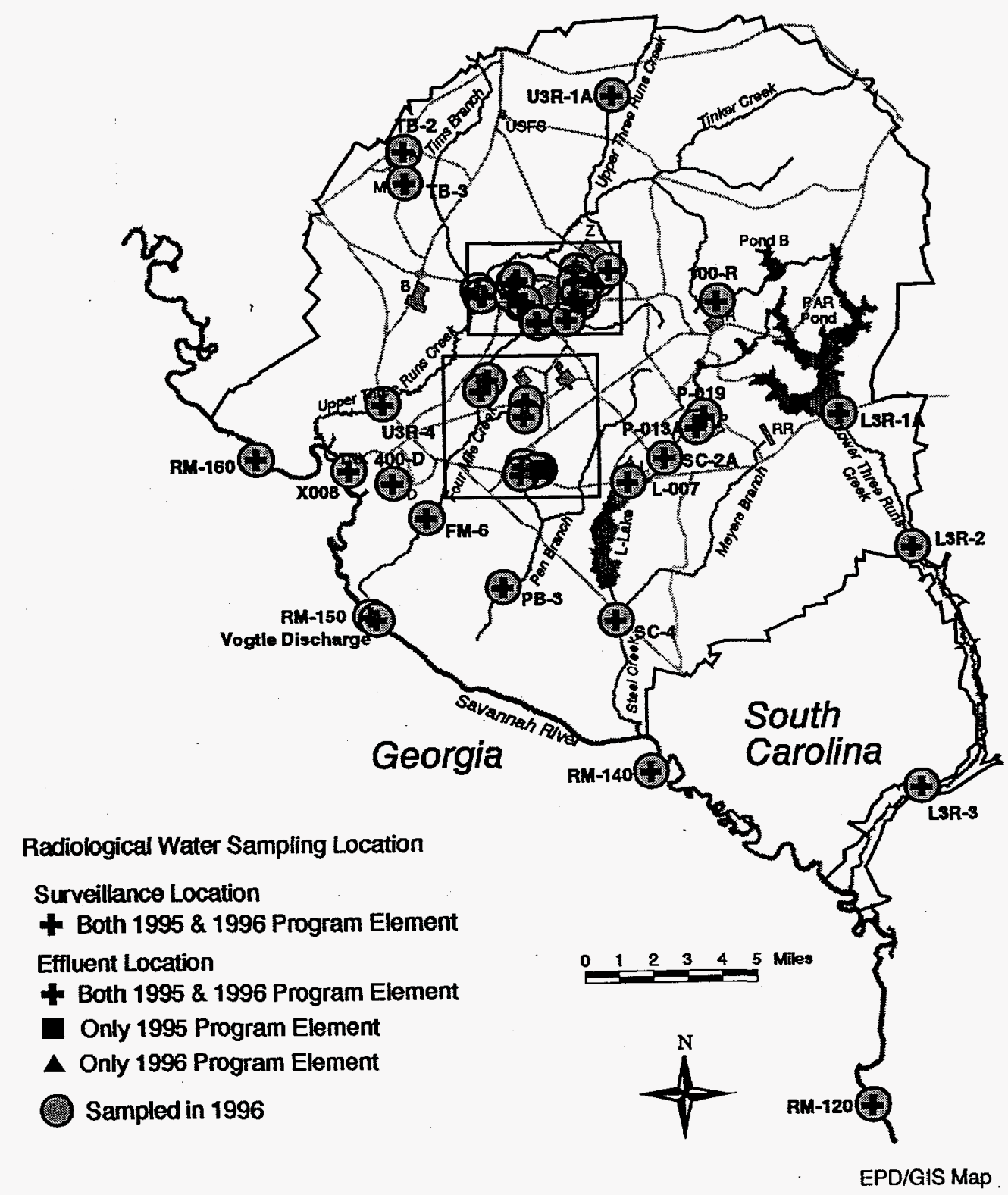




\section{Figure 2 Aerial Photograph, Thermal Infrared Image At High River Stage}

This figure shows the flow pattern of hot-water discharge from Four Mile Creek and Pen Branch into the Savannah River Swamp. The left side of the figure shows the discharge from Four Mile Creek and from Pen Branch. The right side of the figure shows (1) the adjoining downstream section of the swamp and (2) how the heated water from Four Mile Creek and Pen Branch maintains its identity close to the bank for about 5 miles. The lower edge of the right-hand panel shows where the swamp narrows at the Steel Creek Landing. Note that the hot water's thermal infrared signature does not enter the river's main channel until some point downstream of the mouth of Steel Creek.

This image was duplicated from A Thermal Infrared Survey of the Savannah River Plant, Aiken, South Carolina, Winter Survey, DOE/ONS-8317. The survey was conducted March 12, 1983. The report was issued by EG\&G Energy Measurements in December 1983. 


\section{SAVANNAH RIVER PLANT}

\section{MARCH 12, 1983}

COMPOSITE IMAGE OF SAVANNAH RIVER AND SWAMP

UPPER SECTION
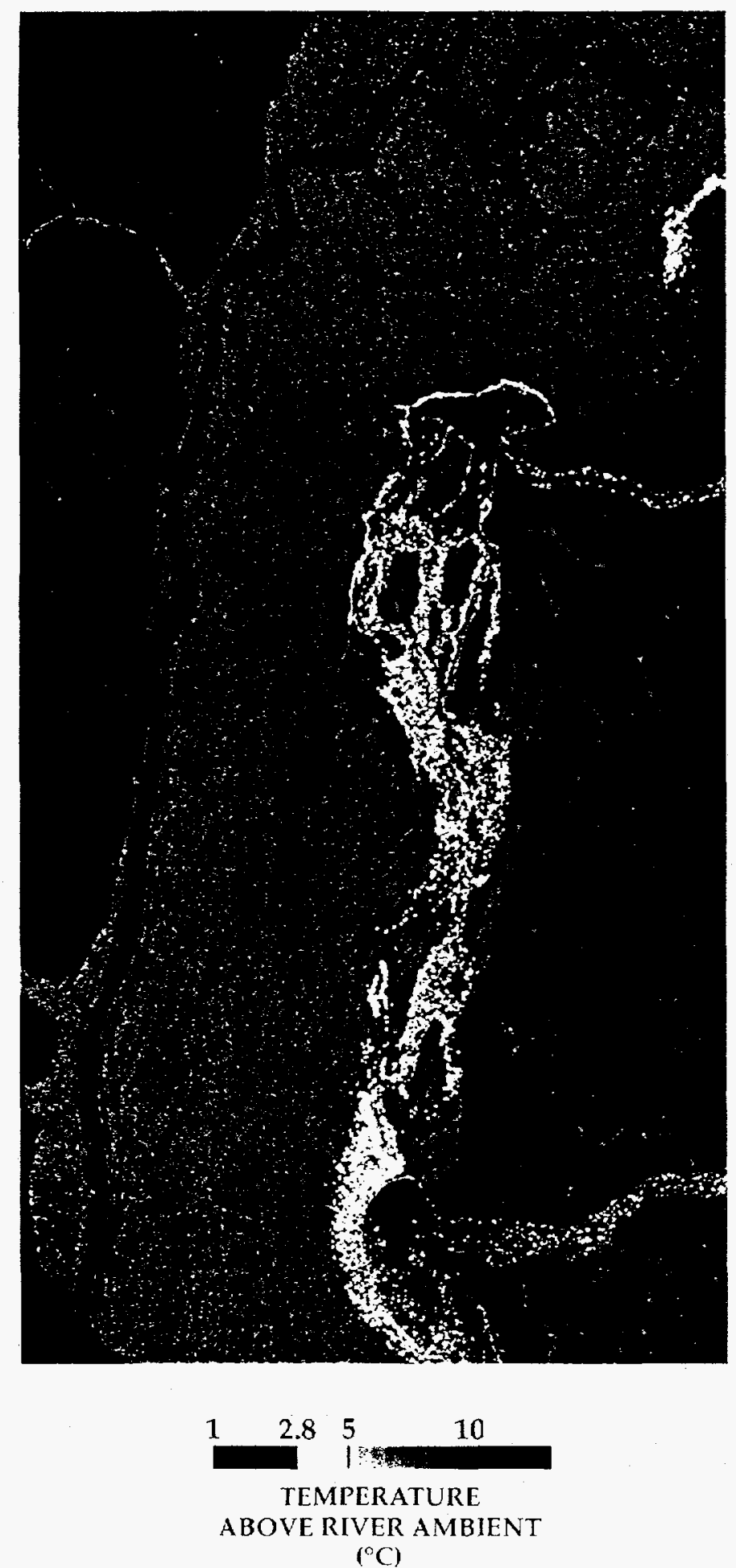

LOWER SECTION
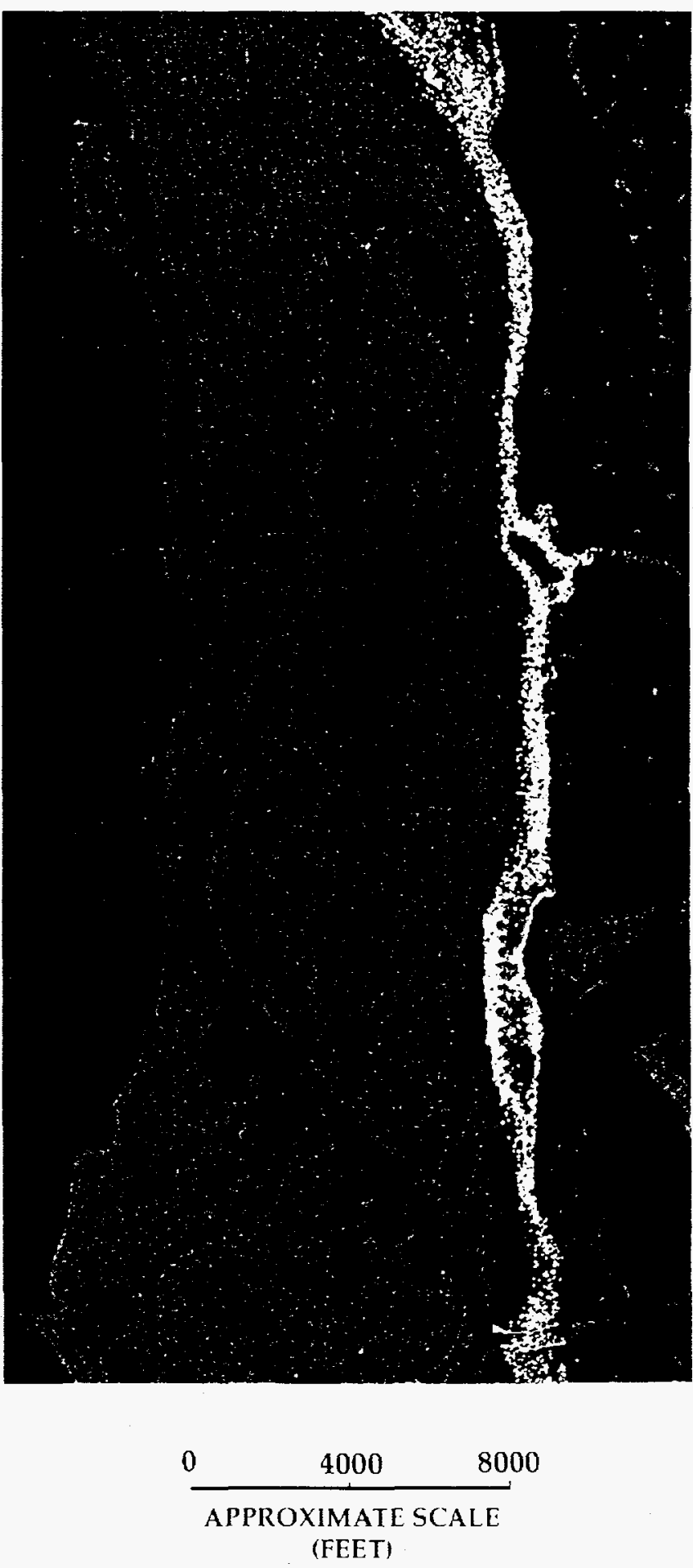

Figure 2 Aerial Photograph, Thermal Infrared Image at High River Stage 
Figure 3 Aerial Photograph, Thermal Infrared Image At Low River Stage

This figure shows the flow pattern of hot-water discharge from Four Mile Creek into the Savannah River. The left side of the figure shows the discharge from Four Mile Creek on March 28, 1981. The right side of the figure shows the same area in August 1981. Note that the discharge from Four Mile Creek enters the main channel of the Savannah River and mixes enough to lose its temperature differential in a few thousand feet.

This image was duplicated from A Study of Thermal Plumes at the Savannah River Plant, Aiken, South Carolina, EGG-1183-1827. The survey was conducted in 1981. The report was issued by EG\&G Energy Measurements in June 1982. 


\section{SAVANNAH RIVER PLANT}

COMPARISON OF THE THERMAL PLUME FROM FOUR MILE CREEK IN MARCH AND AUGUST 1981

MARCH 28, 1981

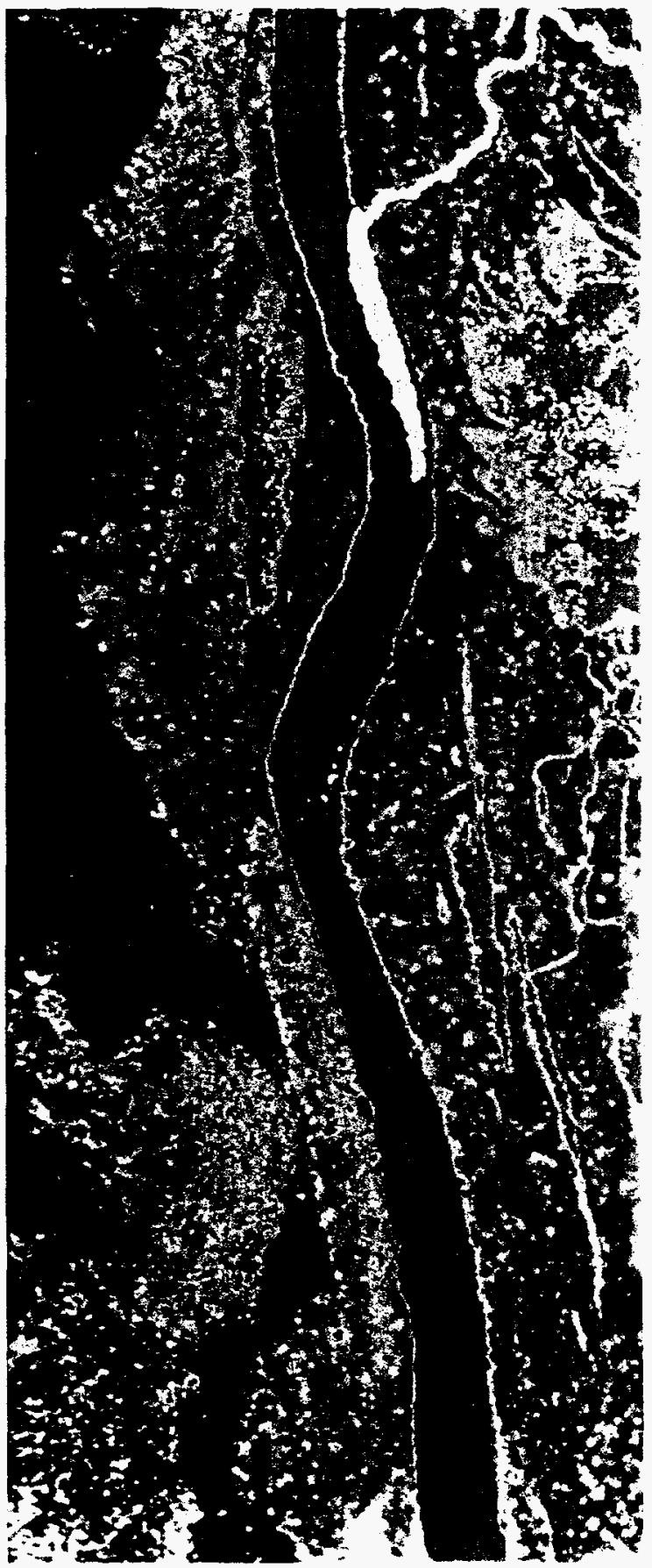

O 2.8

TEMPERATURE

ABOVE RIVER AMBIENT

$\left({ }^{\circ} \mathrm{C}\right)$
AUGIST 25, 1981

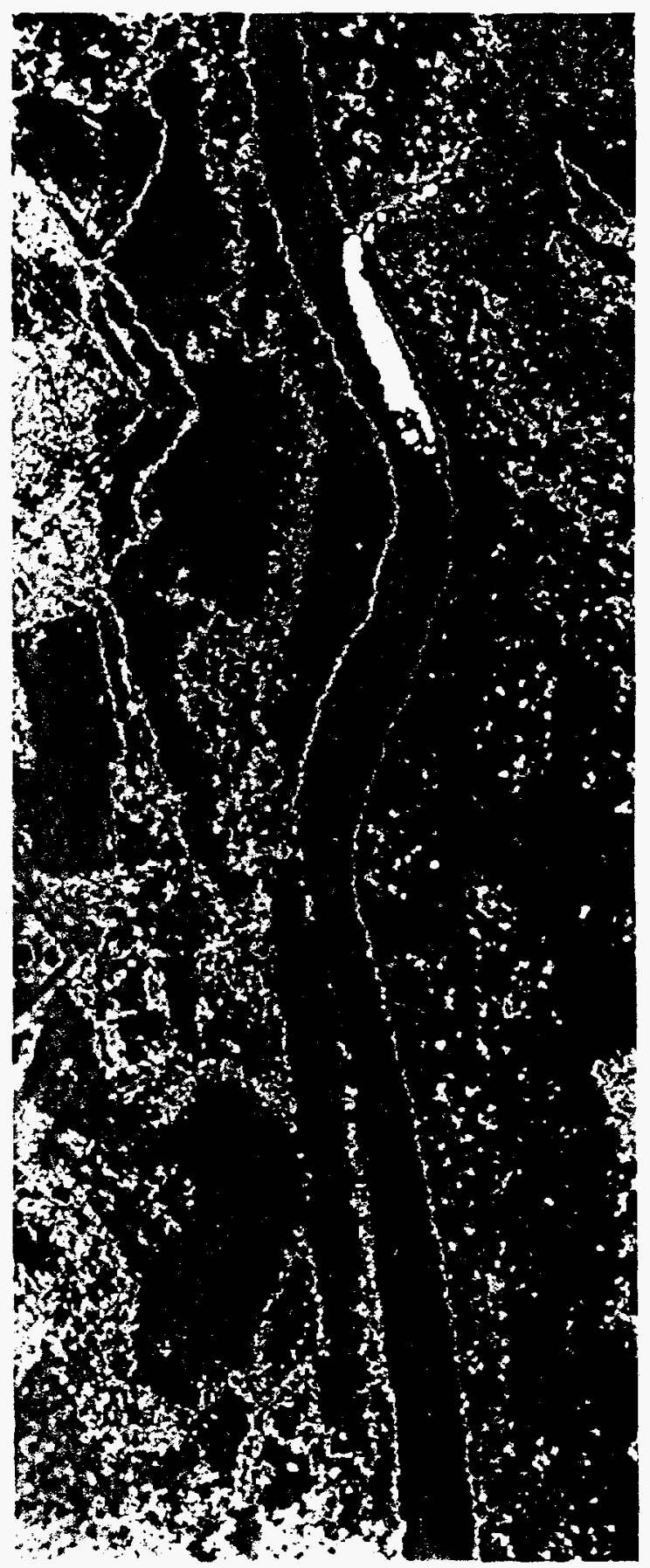

$\underbrace{0 \quad 2000}_{\substack{\text { APPROXIMATE SCALE } \\ \text { (FEET) }}}$

Figure 3 Aerial Photograph, Thermal Infrared Image at Low River Stage 
Figure 4 Tritium Concentration Along Steel Creek Landing Road During Flood Stage

This figure, supplied by David Hayes of the Savannah River Technology Center, shows tritium concentrations at various points along the road to Steel Creek Landing while the Savannah River is at flood stage. The distance reference point (" 0 " on the horizontal axis) is the location of the bank at low flow. Increasing distance shows landward distance from the "normal" bank. At flood stage, the water's edge is about 1600 feet up the road from the "normal" water's edge. Note that tritium is nominally absent from the water except in the 600 feet closest to the water's edge.

\section{Tritium Concentration Along Steel Creek Landing Road During Flood Stage, 2/28/90}

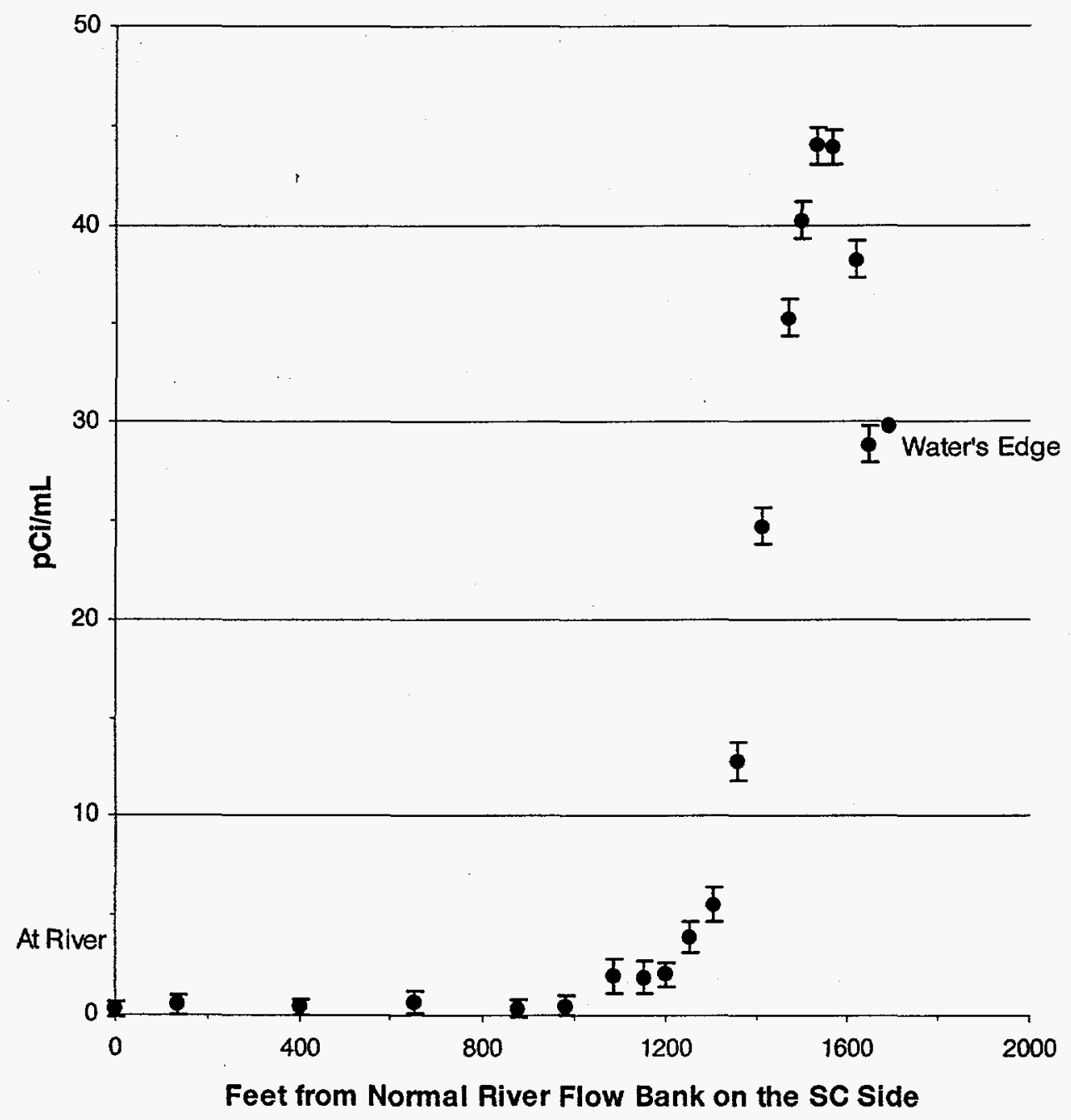


Figure 5 River Stage from January 1997 to January 1998

This figure shows changes in river levels from January 1997 to January 1998. Data are supplied through the U.S. Army Corps of Engineers Website. Note that river stage was at its highest in March and December and also was high in May. SCDHEC's weekly grab samples showed elevated tritium concentrations at Steel Creek Landing February 18 and 25, March 4 and 11, May 6, and December 30. EMS weekly composite samples from River Mile 120 show below-average concentrations for those weeks.

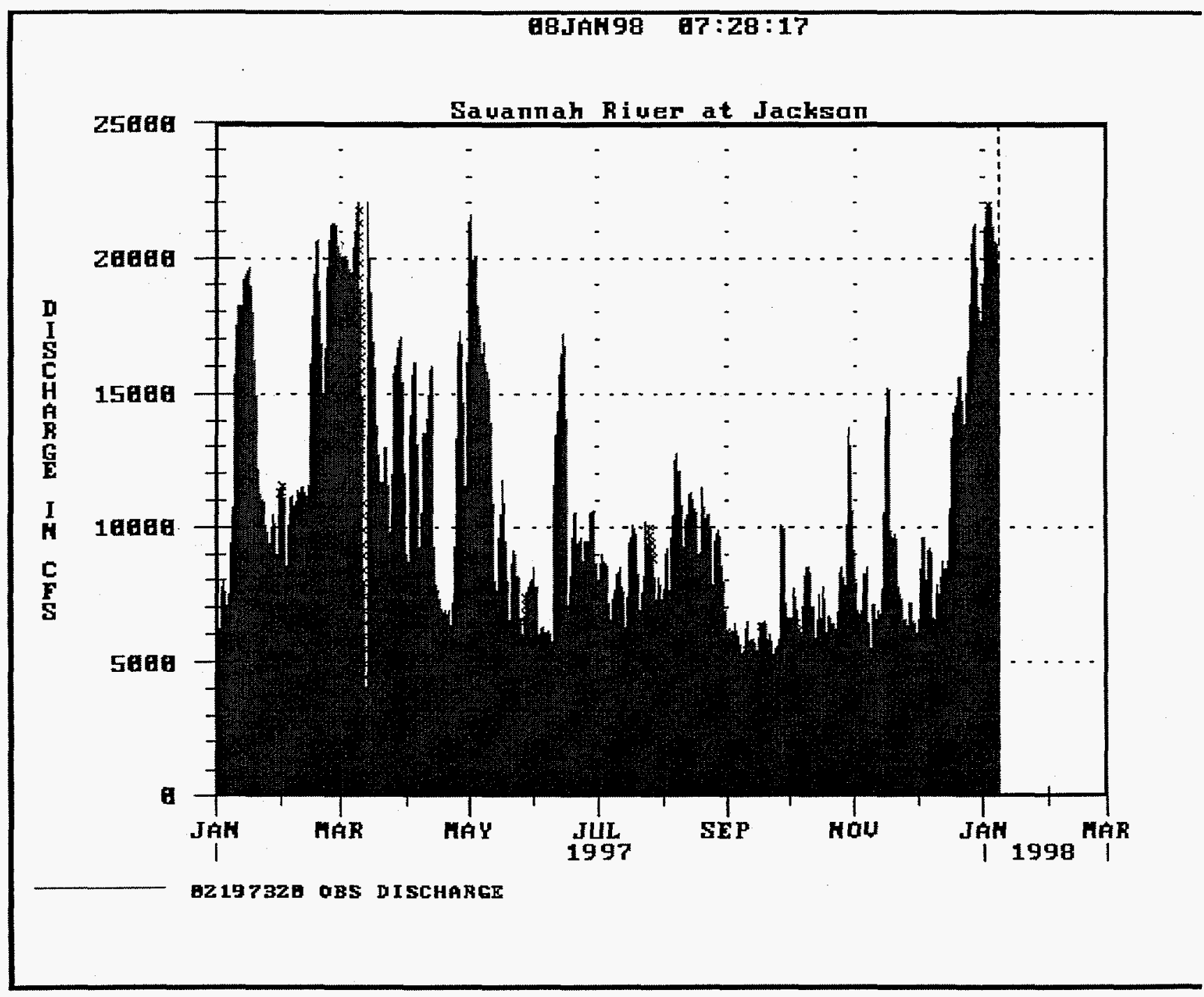


Table 1 Tritium Concentrations in the Savannah River at River Mile 140 (Steel Creek)

Note that these are 1997 weekly composite samples. Steel Creek itself had a fairly stable concentration of tritium throughout the year, ranging from 4 to $8 \mathrm{pCi} / \mathrm{mL}$ in SCDHEC's weekly samples. An asterisk in the "Stop Sample" column denotes a grab sample rather than a composite.

\begin{tabular}{|c|c|c|c|c|c|c|c|}
\hline $\begin{array}{l}\text { Tracking } \\
\text { Number }\end{array}$ & $\begin{array}{l}\text { Start } \\
\text { Sample }\end{array}$ & $\begin{array}{l}\text { Stop } \\
\text { Sample }\end{array}$ & $\begin{array}{l}\text { Sample } \\
\text { Days }\end{array}$ & $\begin{array}{l}\text { Activity } \\
(\mathrm{pCi} / \mathrm{L})\end{array}$ & $\begin{array}{l}\text { Sigma } \\
(\mathrm{pCi} / \mathrm{L})\end{array}$ & $\begin{array}{l}\text { Eff Vol } \\
\text { (L) }\end{array}$ & $\begin{array}{l}\text { Release } \\
\text { (Curies) }\end{array}$ \\
\hline 97000485 & 31DEC96 & 07JAN9 7 & 7 & $2.10 \mathrm{E}+03$ & $1.47 \mathrm{E}+02$ & $*$ * NA * * & $\star * N A * *$ \\
\hline 97000486 & 07JAN97 & 14 JAN9 7 & 7 & $1.48 \mathrm{E}+03$ & $1.43 E+02$ & $\star * N A * *$ & $\star * N A * *$ \\
\hline 97000487 & 14 JAN9 7 & 21JAN97 & 7 & $1.31 \mathrm{E}+03$ & $1.41 \mathrm{E}+02$ & $\star * N A * *$ & $\star * N A * *$ \\
\hline 97000488 & 21JAN97 & 28JAN97 & 7 & $1.59 \mathrm{E}+03$ & $1.43 \mathrm{E}+02$ & $\star * \mathrm{NA} * *$ & $\star \star N A * \star$ \\
\hline 97808088 & 04FEB97 & 04 FEB9 7 * & * 0 & 1. $32 \mathrm{E}+03$ & 1. $42 \mathrm{E}+02$ & $\star * N A * *$ & $* * N A * *$ \\
\hline 97001673 & 04 FEB9 7 & 11FEB97 & 7 & 1. $32 \mathrm{E}+03$ & 1. $40 \mathrm{E}+02$ & $\star * N A * *$ & $\star \star N A * *$ \\
\hline 97001674 & 11FEB97 & 19FEB97 & 8 & $1.21 \mathrm{E}+03$ & $1.48 \mathrm{E}+02$ & $\star * N A * *$ & $\star * N A * *$ \\
\hline 97001675 & 19FEB97 & $25 \mathrm{FEB} 97$ & 6 & $1.14 \mathrm{E}+03$ & $1.49 E+02$ & $\star * N A \star *$ & $\star * N A * *$ \\
\hline 97002324 & 25 FEB 97 & 04MAR97 & 7 & $4.33 E+02$ & $1.40 E+02$ & $* * \mathrm{NA} * *$ & $* * N A * *$ \\
\hline 97002325 & 04MAR97 & 11MAR97 & 7 & $4.51 \mathrm{E}+02$ & $1.41 \mathrm{E}+02$ & $* * \mathrm{NA} * *$ & $\star * N A * *$ \\
\hline 97002326 & IIMAR97 & 18MAR97 & 7 & $5.06 \mathrm{E}+02$ & 1. $36 \mathrm{E}+02$ & $\star * N A * *$ & $* \star N A * *$ \\
\hline 97002327 & 18MAR97 & 25MAR97 & 7 & $9.84 \mathrm{E}+02$ & $1.45 \mathrm{E}+02$ & **NA** & $\star * N A * *$ \\
\hline 97003322 & 25MAR97 & O1APR97 & 7 & $1.20 \mathrm{E}+03$ & $1.37 \mathrm{E}+02$ & **NA $* *$ & **NA * * \\
\hline 97003323 & 01APR97 & 08APR97 & 7 & $1.18 \mathrm{E}+03$ & $1.38 \mathrm{E}+02$ & $\star * \mathrm{NA} * *$ & $\star * N A * *$ \\
\hline 97003324 & 08APR97 & 15APR97 & 7 & $\mathrm{E}+02$ & $1.34 \mathrm{E}+02$ & $\star * N A * *$ & $\star * N A * *$ \\
\hline 97003325 & 15APR97 & 22APR97 & 7 & $1.59 \mathrm{E}+03$ & $1.38 \mathrm{E}+02$ & **NA** & $\star * N A * *$ \\
\hline 97003326 & 22APR97 & 29APR97 & 7 & $1.21 \mathrm{E}+03$ & $1.38 \mathrm{E}+02$ & * *NA * * & $\star * N A * *$ \\
\hline 97004242 & 29APR97 & 06MAY97 & 7 & $1.09 \mathrm{E}+03$ & $1.38 E+02$ & $* * N A * *$ & $* * N A * *$ \\
\hline 97004243 & 06 MAY97 & 13MAY97 & 7 & $1.05 \mathrm{E}+03$ & $1.37 \mathrm{E}+02$ & $\star * N A * *$ & $* * N A * *$ \\
\hline 97004244 & 13MAY97 & 20МАY97 & 7 & $9.82 \mathrm{E}+02$ & $1.40 \mathrm{E}+02$ & $\star *$ NA $* *$ & $\star * N A * \star$ \\
\hline 97004245 & 20MAY97 & 28MAY97 & 8 & $5 E+02$ & $6 \mathrm{E}+02$ & $\star \star N A * \star$ & $* * N A * *$ \\
\hline 97005624 & 28MAY97 & 03JUN97 & 6 & $1: 8$ & 1.4 & $\star \star N A \star \star$ & $* * \mathrm{NA} * *$ \\
\hline 97005625 & 03 JUN97 & 10JUN9 7 & 7 & $2.88 \mathrm{E}+03$ & $9.36 \mathrm{E}+01$ & $\star * N A * *$ & $* * N A * *$ \\
\hline 97005626 & 10JUN97 & 17JUN97 & 7 & $2.43 \mathrm{E}+03$ & $1.48 \mathrm{E}+02$ & * *NA * * & $\star \star N A * *$ \\
\hline 97810279 & 24 JUN97 & 24 JUN9 7 * & * 0 & $7.31 \mathrm{E}+02$ & 1. $36 \mathrm{E}+02$ & $\star * N A * *$ & $* * N A * *$ \\
\hline 97006557 & 24 JUN97 & 01JUL97 & 7 & $1.02 \mathrm{E}+03$ & $4 \mathrm{E}+02$ & $\star * N A * *$ & $\star * N A * *$ \\
\hline 97006558 & 01JUL97 & 09JUL97 & 8 & & & $\star * N A * *$ & $\star * N A * *$ \\
\hline 97006559 & 09JUL97 & $15 \mathrm{JUI}$ & 6 & $1 E+03$ & 1.4 & $\star * N A * *$ & $* * \mathrm{NA} * *$ \\
\hline & 15JUL97 & 22JUL97 & 7 & $1.73 \mathrm{E}+03$ & $1.37 \mathrm{E}+02$ & $\star * N A * *$ & * *NA ** \\
\hline 97006561 & 22JUL97 & 29JUL97 & 7 & $1.08 \mathrm{E}+03$ & 1. $37 \mathrm{E}+02$ & $\star *$ NA $* *$ & $\star * N A * *$ \\
\hline 97006951 & 29JUL97 & 05AUG97 & 7 & $9.69 \mathrm{E}+02$ & $1.37 \mathrm{E}+02$ & $\star * \mathrm{NA} * *$ & **NA ** \\
\hline & 05AUG97 & 12AUG97 & 7 & $1.03 E+03$ & 1. $37 E+02$ & * *NA * * & $\star * N A * *$ \\
\hline & 12AUG9 7 & 19AUG97 & 7 & $1.10 E+03$ & 1. $38 E+02$ & $\star * N A * *$ & $\star * N A * *$ \\
\hline & 19AUG97 & 26AUG97 & 7 & $1.32 \mathrm{E}+03$ & $1.40 \mathrm{E}+02$ & $\star * N A * *$ & $\star *$ NA $* *$ \\
\hline & 26AUG97 & 02SEP97 & 7 & $1.06 \mathrm{E}+03$ & $1.11 \mathrm{E}+02$ & $\star \star N A * *$ & $* * \mathrm{NA} * *$ \\
\hline 97008872 & 02SEP97 & 09SEP97 & 7 & 1.42 & $1.14 \mathrm{E}+02$ & $\star \star N A * *$ & **NA ** \\
\hline 97008873 & 09SEP97 & 16SEP97 & 7 & $1.52 \mathrm{E}+03$ & $1.14 \mathrm{E}+02$ & $\star * N A * *$ & $\star * N A * *$ \\
\hline & 16SEP9 7 & 23SEP97 & 7 & $1.81 \mathrm{E}+03$ & & $\star \star N A * *$ & $\star * N A * *$ \\
\hline 97808520 & 30SEP97 & 30SEP97* & * 0 & $1.60 \mathrm{E}+03$ & $1.48 \mathrm{E}+02$ & $\star * N A * *$ & **NA ** \\
\hline & 30SEP97 & 07ОСТ97 & 7 & $8.59 \mathrm{E}+02$ & $1.42 \mathrm{E}+02$ & $\star * N A * *$ & **NA $* *$ \\
\hline & 07ОСТ97 & $140 \mathrm{OT} 97$ & 7 & $1.15 \mathrm{E}+03$ & $1.38 \mathrm{E}+02$ & $\star * N A * *$ & $* * N A * *$ \\
\hline & 14 ОСТ97 & 210 CT97 & 7 & $1.33 \mathrm{E}+03$ & $1.15 \mathrm{E}+02$ & $\star *$ NA * * & $\star * N A * *$ \\
\hline & 210Ст97 & $280 \mathrm{CT} 97$ & 7 & $1.57 \mathrm{E}+03$ & $1.17 \mathrm{E}+02$ & $\star N$ NA * * & $\star \star N A * *$ \\
\hline & $280 C T 97$ & 04 NOV9 7 & 7 & $1.45 \mathrm{E}+03$ & $1.47 \mathrm{E}+02$ & $\star * N A * *$ & $\star \star N A * *$ \\
\hline 97010868 & 04 NOV97 & 11NOV9 7 & 7 & 1. $35 \mathrm{E}+03$ & $1.16 \mathrm{E}+02$ & $\star * N A * *$ & $\star \star N A * *$ \\
\hline & 11NOV97 & 18NOV9 7 & 7 & 1. $22 \mathrm{E}+03$ & $1.15 \mathrm{E}+02$ & **NA * * & $\star \star N A \star *$ \\
\hline 97010870 & 18 NoV9 7 & 25 NOV9 7 & 7 & 1. $39 \mathrm{E}+03$ & $1.15 \mathrm{E}+0.2$ & $\star * N A * *$ & $\star * \mathrm{NA} * *$ \\
\hline 97011826 & 25 NOV9 7 & $02 D E C 97$ & 7 & $1.65 \mathrm{E}+03$ & $1.17 \mathrm{E}+02$ & * *NA * * & * *NA * * \\
\hline 97011827 & $02 \mathrm{DEC} 97$ & 09DEC97 & 7 & $1.43 \mathrm{E}+03$ & $1.17 \mathrm{E}+02$ & * *NA * * & $\star \star N A * *$ \\
\hline 97011828 & 09DEC97 & $16 \mathrm{DEC} 97$ & 7 & $2.02 \mathrm{E}+03$ & 1. $21 \mathrm{E}+02$ & $\star * N A * *$ & $\star * N A * *$ \\
\hline 97011829 & 16DEC97 & 23DEC 97 & 7 & $7.65 E+02$ & $1.18 E+02$ & $\star * N A * *$ & $\star * N A * *$ \\
\hline 97011830 & 23 DEC 97 & $30 \mathrm{DEC} 97$ & 7 & 1. $28 \mathrm{E}+03$ & $1.21 \mathrm{E}+02$ & $\star \star N A * *$ & **NA $* *$ \\
\hline
\end{tabular}


Table 2 Tritium Concentrations in the Savannah River at River Mile 120 (Highway 301 Bridge)

Note that these are 1997 weekly composite samples. SCDHEC grab samples showed elevated tritium concentrations at Steel Creek Landing (March, May, December) when the downstream concentrations were low, as would be expected during times of high river flow.

\begin{tabular}{|c|c|c|c|c|c|c|c|}
\hline $\begin{array}{l}\text { Tracking } \\
\text { Number }\end{array}$ & $\begin{array}{l}\text { Start } \\
\text { Sample }\end{array}$ & $\begin{array}{l}\text { Stop } \\
\text { Sample }\end{array}$ & $\begin{array}{l}\text { Sample } \\
\text { Days }\end{array}$ & $\begin{array}{l}\text { Activity } \\
\text { (pCi/L) }\end{array}$ & $\begin{array}{l}\text { Sigma } \\
(\mathrm{pCi} / \mathrm{L})\end{array}$ & $\begin{array}{l}\text { Eff Vol } \\
\text { (L) }\end{array}$ & $\begin{array}{l}\text { Release } \\
\text { (Cúries) }\end{array}$ \\
\hline 97000429 & & & 7 & $1.64 \mathrm{E}+03$ & $1.45 \mathrm{E}+02$ & $1.02 \mathrm{E}+11$ & \\
\hline & 7JAN9 7 & IJAN97 & 7 & & & & \\
\hline 97000431 & 14 JAN97 & 1JAN97 & 7 & $25 E+02$ & $.80 \mathrm{E}+01$ & $3.14 E+11$ & $2.90 \mathrm{E}+02$ \\
\hline & 21JAN97 & 28JAN97 & 7 & & $1.41 \mathrm{E}+02$ & $1.83 \mathrm{E}+11$ & $2.74 \mathrm{E}+02$ \\
\hline 97001618 & 28JAN97 & 04FEB97 & 7 & $.06 \mathrm{E}+03$ & $1.41 \mathrm{E}+02$ & $1.54 \mathrm{E}+11$ & \\
\hline 97001619 & 04 FEB97 & 11 FEB97 & 7 & $9.42 \mathrm{E}+02$ & $1.39 \mathrm{E}+02$ & $1.72 \mathrm{E}+11$ & $1.62 \mathrm{E}+02$ \\
\hline 97001620 & 11FEB97 & 19 FEB97 & 8 & & $1.03 \mathrm{E}+02$ & & $2.14 \mathrm{E}+02$ \\
\hline & 19FEB97 & 25 FEB 97 & 6 & & & & \\
\hline 97002264 & 25FEB97 & 04MAR97 & 7 & $6.26 \mathrm{E}+02$ & $1.42 \mathrm{E}+02$ & $3.88 \mathrm{E}+11$ & $2.43 \mathrm{E}+02$ \\
\hline 97002265 & 04MAR97 & 11MAR97 & 7 & & & $E+11$ & +02 \\
\hline 97002 & 97 & $18 \mathrm{MA}$ & 7 & & & $4.27 \mathrm{E}$ & +02 \\
\hline & & & 7 & & & & \\
\hline & 25MAR97 & $01 \mathrm{~A}$ & 7 & & & 2.4 & +02 \\
\hline & & & 7 & & & & \\
\hline 97003257 & 08APR97 & 15APR9 7 & 7 & & & & +02 \\
\hline 97003258 & 15APR97 & 22APR9 7 & 7 & +02 & & 1.1 & -02 \\
\hline & & & 7 & & & 1.9 & \\
\hline & 97 & 061 & 7 & & & & \\
\hline & 06 MAY97 & 13MAY97 & 7 & & & 2.8 & \\
\hline & 13MAY97 & 20MAY97 & 7 & & & & \\
\hline 970 & 20MA & $28 \mathrm{r}$ & 8 & & & & \\
\hline & $28 \mathrm{M}$ & & 6 & & & 1.0 & -02 \\
\hline & & & 7 & & & & \\
\hline 9700 & $10 \pi$ & $17 \mathrm{~J}$ & 7 & & & 2.1 & 01 \\
\hline & & & 7 & & & & \\
\hline & & & 7 & & & & \\
\hline & $01 \mathrm{~J}$ & & 8 & & & 1.4 & \\
\hline & & & 6 & & & & \\
\hline $97 C$ & $15 \mathrm{~J}$ & $22 i$ & 7 & & & 1.3 & +02 \\
\hline 505 & & & 7 & & 9.7 & 1.3 & 02 \\
\hline & & & 7 & & & & \\
\hline & $05 \mathrm{AT}$ & $12 \mathrm{~A}$ & 7 & & 1.3 & 1.6 & \\
\hline & & & 7 & & & & \\
\hline & & & 7 & & & & \\
\hline & $26 \mathrm{~A}$ & 025 & 7 & & 1.1 & 1.2 & 1.7 \\
\hline & & & 7 & & & & \\
\hline & 09SEP97 & & 7 & & & & \\
\hline & 16 SEP9 7 & $23 \mathrm{~s}$ & 7 & 1.8 & 1.1 & 8.8 & +02 \\
\hline & & & 7 & & & & \\
\hline & 30SEP97 & 07ОСТ97 & 7 & & & & \\
\hline & & $140 \mathrm{CT} 97$ & 7 & & & & \\
\hline & & & 7 & & & & \\
\hline & 210ст97 & 280СТ97 & 7 & & 8.1 & 1.1 & \\
\hline & $280 \mathrm{CT} 97$ & 04 NOV97 & 7 & $1.01 \mathrm{E}+03$ & 1.44 & $1.46 \mathrm{E}+11$ & $1.47 E+02$ \\
\hline & & & 7 & & & & \\
\hline & & & 7 & & & & 1.5 \\
\hline 97010816 & 18NOV97 & 25NOV9 7 & 7 & $1.11 \mathrm{E}+03$ & $1.13 \mathrm{E}+02$ & 1. $29 \mathrm{E}+11$ & $1.44 \mathrm{E}+02$ \\
\hline & 25NOV97 & 02DEC97 & 7 & & & & \\
\hline & $02 D E C 97$ & 09DEC97 & 7 & & $1.14 \mathrm{E}+02$ & & 1. $38 \mathrm{E}+02$ \\
\hline & 09DEC97 & 16DEC97 & 7 & & 1. $16 \mathrm{E}+02$ & $1.39 \mathrm{E}+11$ & $1.91 \mathrm{E}+02$ \\
\hline & & 23DEC 97 & 7 & & & $2.35 \mathrm{E}+11$ & \\
\hline 97011 & & & & & $1.19 \mathrm{E}+02$ & 3. $31 E+11$ & $3.20 \mathrm{E}+02$ \\
\hline
\end{tabular}




\section{Attachment 1 SCDHEC Analytical Data from Weekly Grab Samples}

This table was provided by Michael Moore of SCDHEC. Location SAV-23 is the station of interest. A map identifying the sampling station locations is provided at the back of this attachment. Note that the highest concentrations seen at SAV-23 coincide with dates of high river stage (figure 5). 


\section{Radiological Surface Water Tritium Data ( $\mathrm{pCi} / \mathrm{L}$ )}

\begin{tabular}{|c|c|c|c|c|c|c|c|c|c|c|c|}
\hline $\begin{array}{l}\text { Collected } \\
\text { UTR-10 }\end{array}$ & $\frac{02 / 0.4 / 97}{432.84}$ & $\frac{02 / 11 / 97}{438.08}$ & $\frac{02 / 18 / 97}{653.70}$ & $\frac{102 / 25 / 97}{361.93}$ & $\frac{0.310419 / 2}{589.15}$ & $\frac{03 / 1 / 1 / 97}{571.32}$ & $\frac{03198197}{552.82}$ & $\frac{03 / 251.97}{492.93}$ & $\frac{0.4 / 01197}{510.73}$ & $\frac{354.56}{354.56}$ & $\frac{04125197}{417.92}$ \\
\hline $\mathrm{SAV}=24$ & $<238$ & $<238$ & +rit 24877 & $3+37264$ & $\angle 229$ & $2231 ;$ & 14229 & 27230 & 24255 & $<221$ & $<233$ \\
\hline \multicolumn{12}{|l|}{ FMB-07 } \\
\hline UTR-08 & 37298.98 & 28302.13 & 11753.80 & 1641647 & 1725771 & 13935 & 125968 & 41275.31 & 1318.66 & 12056.98 & 113492 \\
\hline SAV-02 & 277.76 & 851.33 & 631.80 & 523.81 & 260.22 & 263.27 & 644.62 & $<230$ & 240.86 & 758.51 & $<233$ \\
\hline FMB-03 & 226626.59 & 24575106 & 17563812 & 216033.08 & 25359187 & 276642.96 & 27339872 & 24936374 & 24388069 & 25435996 & 27738496 \\
\hline PNB-03 & 138323.13 & 160445.67 & 93038.44 & 94050.39 & 112625.25 & 127468.05 & 123572.14 & 140605.71 & 143215.88 & 153274.95 & 153730.40 \\
\hline $\mathrm{STCl}_{04}$ & 8155.11 & 818196 & 785404 & 7736.51 & 791695 & 765748 & 7523.43 & 7407.48 & 706710 & 705929 & 7399.82 \\
\hline SAV-23 & 2853.59 & 1811.54 & 12666.98 & 15833.32 & 24286.56 & 20942.55 & 3731.16 & 1154.68 & 3004.28 & 1529.70 & 3259.39 \\
\hline SAV05 & 1454.69 & +46731 & i'79877 & 1919.89 & 1132843 & 1765775 & 1961447 & 109719 & 2009.71 & 44474 & $<233$ \\
\hline SAV-08 & 910.46 & 1024.80 & 981.54 & 1015.48 & 455.73 & 565.77 & 696.44 & 948.94 & 1622.59 & 1270.58 & 1450.56 \\
\hline LTR-02 & 3172.75 & 83565 & 1006,36 & 87734 & 870,33 & 88231 & 175220 & 776192 & 81961 & 101088 & 1148.05 \\
\hline SAV-25 & & 872.91 & & & & 499.80 & & & & & 1118.89 \\
\hline \multicolumn{12}{|l|}{$S V / 355 \mathrm{k}$} \\
\hline SV-191 & & & & & & & & & & 1250.98 & \\
\hline $\mathrm{SV}=356$ & & & & & 3 & & & & & & \\
\hline \multicolumn{12}{|l|}{ SAV-08C } \\
\hline LTR-02C & & & & & +2 & & & & & & \\
\hline FMB-03C & & & & & & & & & & & \\
\hline
\end{tabular}




\section{Radiological Surface Water Tritium Data (pCi/L)}

\begin{tabular}{|c|c|c|c|c|c|c|c|c|c|c|c|}
\hline Collected & $04 / 23 / 97$ & $04 / 29 / 97$ & $05 / 06 / 97$ & $05 / 13 / 97$ & $05 / 20 / 97$ & $05 / 27 / 97$ & $06 / 03 / 97$ & $06 / 10 / 97$ & $06 / 17: 197$ & $06 / 24 / 97$ & $07101 / \mathrm{s}$ \\
\hline UTR-10 & 801.57 & 560.05 & 431.37 & 475.30 & 462.70 & 366.44 & 562.85 & 288.33 & 474.17 & 440.38 & $<239$ \\
\hline SAV-24 & 316.97 & 24638 & $<223$ & $<225$ & 230 & (1) 229 & 402217 & $<227$ & 4218 & $<221$ & $<239$ \\
\hline FMB-07 & & & & & & & & & & & \\
\hline UTR-08 & 12567.08 & 248265 & 1632.01 & 480236 & 765.16 & 940.61 & 90146 & 933.70 & 106773 & 1146.75 & 843.32 \\
\hline SAV-02 & 389.72 & 566.33 & 404.40 & 434.82 & $<230$ & 290.44 & $<221$ & $<227$ & 436.44 & $<221$ & $<239$ \\
\hline$F M B=03$ & 231855.89 & 14695717 & 24735945 & 22515722 & 29550801 & 29841784 & 28570026 & 249068.22 & 21762475 & 26846786 & 212103.91 \\
\hline PNB-03 & 166666.13 & 57319.18 & 132643.35 & 165106.62 & 177593.86 & 159186.21 & 158894.08 & 155664.75 & 156655.33 & 156543.73 & 157614.66 \\
\hline STC:04 & 7131.87 & 6769.82 & 729166 & 5553.51 & 6251.64 & 586144 & 598623 & 5906.32 & 6549.43 & 5666.16 & 5599.68 \\
\hline SAV-23 & 1787.97 & 4669.92 & 14897.57 & 3939.80 & 1218.00 & 1186.08 & 1313.87 & 927.51 & 5576.87 & 1081.25 & 1079.28 \\
\hline SAV 05 & 57796 & 696,33 & 11142.07 & 463523 & 64565 & 83625 & 134880 & 1826.29 & 40941 & 659.45 & 57807 \\
\hline SAV-08 & 1047.98 & 1395.20 & 800.80 & 1258.64 & 680.00 & 881.32 & 1078.76 & 1951.31 & 1745.76 & 1089.13 & 517.25 \\
\hline LTR:02 & 874.96 & 911104 & 101721 & 97102 & 845.14 & 1015.55 & 105020 & 99846 & 965.24 & 808.81 & 811.22 \\
\hline SAV-25 & & & & & & & & & & & \\
\hline SV 355 & Xris & 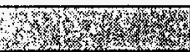 & & & & 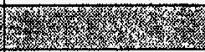 & $y^{2}$ & rex & 3ity & 43275 & 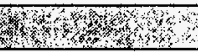 \\
\hline SV-191 & & & & & & & & & & 932.88 & \\
\hline SV-356 & & & 4 & & X & $34 \quad 3$ & 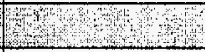 & trus & 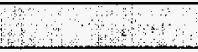 & $<239$ & \\
\hline SAV-08C & & & & & & & & & & & \\
\hline LTR=02C & & & 9 & & 322 & 10 & & $\mathrm{Bu}^{2}$ & & & \\
\hline FMB-03C & & & & & & & & & & & \\
\hline
\end{tabular}




\section{Radiological Surface Water Tritium Data (pCl/L)}

\begin{tabular}{|c|c|c|c|c|c|c|c|c|c|c|c|}
\hline Cóllecte & $07 / 08 / 97$ & $07 / 15 / 97$ & $07 / 22 / 97$ & $07 / 29 / 97$ & $08 / 05 / 97$ & $08 / 12 / 97$ & $08 / 19 / 97$ & $08 / 26 / 97$ & $09 / 02 / 97$ & $09 / 09 / 97$ & $09 / 16 / 97$ \\
\hline UTR-10 & 487.69 & 528.80 & 272.93 & 255.11 & 502.84 & 450.52 & 541.83 & 537.81 & 271.76 & 573.29 & 395.89 \\
\hline SAV-24 & 220.75 & $<219$ & 2919 & 4227 & 74222 & 22443 & 215 & $<217$ & 228 & 23371 & 299.03 \\
\hline FMB-07 & & & & & & & & & & & \\
\hline UTR 08 & 1042.39 & 1218.09 & $\begin{array}{r}999.05 \\
\end{array}$ & 12898.38 & 1054.42 & 142912 & 107128 & 91568 & 907.09 & 822.76 & 925.82 \\
\hline SAV-02 & 373.93 & 296.22 & 235.75 & 779.40 & $<222$ & $<216$ & $<215$ & 274.25 & $<228$ & 351.97 & $<215$ \\
\hline FMB-03 & 248041.04 & 276279.06 & 317538.37 & 30304670 & 320213.37 & 33756046 & 26326919 & 305663.65 & 278313.52 & 330214.17 & 314055.71 \\
\hline PNB-03 & 134610.27 & 128102.51 & 152045.78 & 161340.74 & 177231.11 & 168256.78 & 151517.97 & 174565.19 & 214316.32 & 152452.55 & 193563.05 \\
\hline STC:04 & 5309.94 & $5945: 74$ & 630162 & 609159 & 632400 & 621436 & 34879.01 & 6657.56 & 6777.14 & 7085.55 & 7057.96 \\
\hline$\overline{\text { SAV-23 }}$ & 1688.32 & 1864.03 & 1039.61 & 959.61 & 3177.92 & 806.99 & 839.79 & 818.82 & 1137.46 & 1139.82 & 4896.02 \\
\hline SAV $=05$ & 208703 & 1369.02 & 1309.45 & 1120.67 & 96371 & 105703 & 99806 & 82276 & 1028.19 & 1106.03 & $1726: 62$ \\
\hline SAV-08 & 1080.68 & 1200.64 & 1004.12 & 781.65 & 805.39 & 4529.98 & 1036.92 & 531.05 & 1508.63 & 1081.25 & 811.50 \\
\hline LTR-02 & 96468 & 745.61 & 984.97 & 592.43 & 679.19 & 99734 & 846.54 & 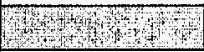 & 848.52 & 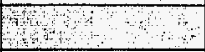 & 1351.00 \\
\hline SAV-25 & & & & & & & & & & & \\
\hline SV 355 w & 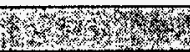 & (3) & & 1460773 & & 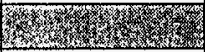 & & 76474 & 1275.95 & 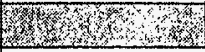 & \\
\hline SV-191 & & & & 876.77 & & & & 585.75 & 1145.20 & & \\
\hline SV-356 & & & & $<222$ & 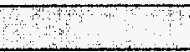 & & the & $<228$ & $<211$ & & \\
\hline SAV-08C & & & & & & & 1031.85 & & 1102.60 & 1117.37 & 1908.88 \\
\hline LTR-02C & & & 4 & Pr & & $m b 1 \mathrm{n}$ & 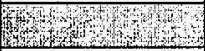 & 1145.45 & 110260 & 131693 & \\
\hline FMB-03C & & & & & & & & & & & \\
\hline
\end{tabular}


Radiological Surface Water Tritium Data (pCi/L)

\begin{tabular}{|c|c|c|c|c|c|c|c|}
\hline \multicolumn{8}{|c|}{ 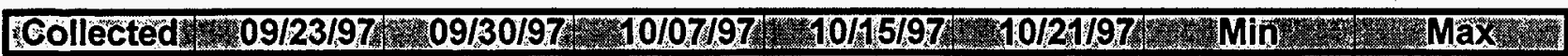 } \\
\hline UTR-10 & 588.49 & 534.71 & 304.95 & 396.76 & 499.51 & $<220$ & 801.57 \\
\hline SAV24 & $\langle 217$ & 30213 & 3226 & (3) & $1<221$ & 14t) 220 & 372.64 \\
\hline FMB-07 & & & 617887.72 & 605428.96 & 412312.97 & 412312.97 & 617887.72 \\
\hline $\operatorname{GTR} 08$ & 661025 & (1) 1363.10 & 4788.13 & 93751 & T. & 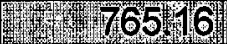 & 37298.98 \\
\hline SAV-02 & 331.70 & 719.99 & $<226$ & 239.66 & 301.29 & $<220$ & 851.33 \\
\hline FMB 03 & a. $=9$ & 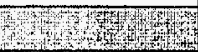 & Fity & 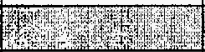 & 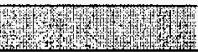 & 44695777 & 337560.46 \\
\hline PNB-03 & 196173.81 & 112670.23 & 169029.14 & 172404.20 & 143126.22 & 57319.18 & 214316.32 \\
\hline STC-04 & 726520 & 656379 & 6934.35 & 672533 & 612594 & 3879.01 & 818196 \\
\hline SAV-23 & 1055.91 & 3384.81 & 936.80 & 1361.62 & 1950.19 & 806.99 & 24286.56 \\
\hline SAV=05 & 1336.35 & 89287 & 6783 & 1471.6 & 73885 & 40220 & 1765775 \\
\hline SAV-08 & 1120.11 & 6027.11 & 735.19 & 758.96 & 1415.76 & 455.73 & 6027.11 \\
\hline HTR 02 & का & मnthy & 737411 & 931.90 & 709.66 & 59243 & 317275 \\
\hline SAV-25 & & & & & & 499.80 & 1118.89 \\
\hline $5 \sqrt{355}$ & 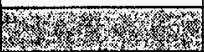 & 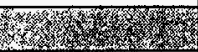 & & 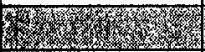 & 187561 & $432 \sqrt{5}$ & \\
\hline SV-191 & & & & & 844.25 & 585.75 & 1250.98 \\
\hline SV -356 & & & & & 222110 & Pr & rix 8220 \\
\hline SAV-08C & 1909.45 & 1261.41 & 985.73 & 967.99 & 1127.91 & 967.99 & 1909.45 \\
\hline LTR $02 C$ & 1135.41 & 1150,35 & Findy & 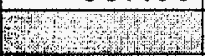 & W & 1102,60 & 131693 \\
\hline FMB-03C & 307871.43 & 200624.10 & 252930.71 & 298429.97 & 237716.31 & 200624.10 & 307871.43 \\
\hline
\end{tabular}




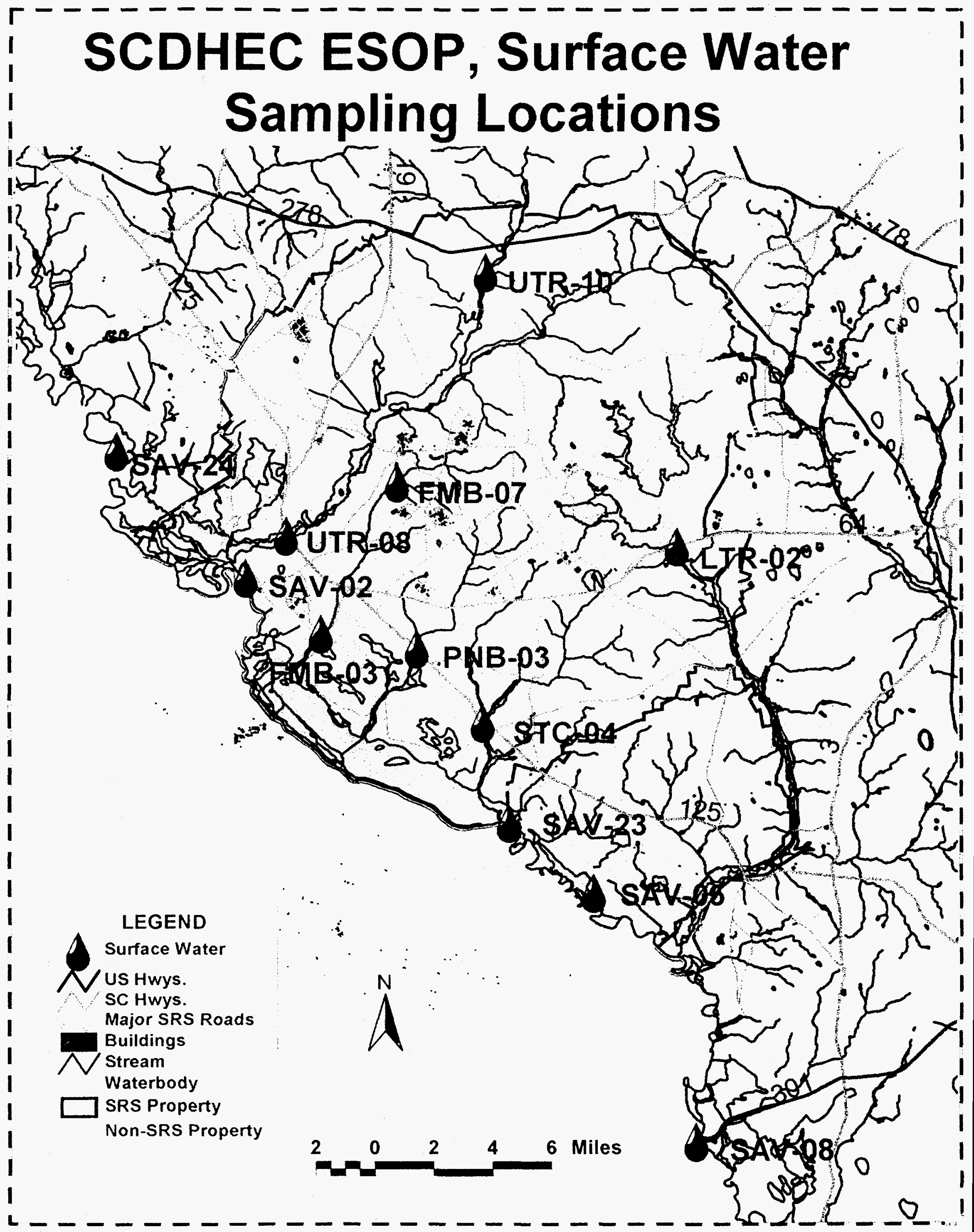

